\title{
AN APPROACH FOR INDOOR WAYFINDING REPLICATING MAIN PRINCIPLES OF AN OUTDOOR NAVIGATION SYSTEM FOR CYCLISTS
}

\author{
A. Makri ${ }^{a}$, S. Zlatanova ${ }^{b}$, E. Verbree $^{c}$ \\ ${ }^{a}$ MSc Geomatics, Faculty of Architecture and the Built Environment, Delft University of Technology, Julianalaan 134, 2628 BL, \\ Delft, The Netherlands, A.Makri@student.tudelft.nl \\ b 3D Geoinformation, Faculty of Architecture and the Built Environment, Department of Urbanism, Delft University of Technology, \\ Julianalaan 134, 2628 BL, Delft, The Netherlands, s.zlatanova@tudelft.nl \\ ${ }^{c}$ GIS Technology, Faculty of Architecture and the Built Environment, OTB Research Institute for the Built Environment, Delft \\ University of Technology, Julianalaan 134, 2628 BL, Delft, The Netherlands, E.Verbree@ tudelft.nl
}

\section{Commissions WG IV/7 and WG V/5}

KEY WORDS: Indoor wayfinding, landmark, signpost, decision point, node network, route graph, Constrained Delaunay Triangulation

\begin{abstract}
:
This work presents an approach to enhance navigation in indoor environments based on a landmark concept. It has already been proved by empirical research that by using landmarks the wayfinding task can be significantly simplified. Navigation based on landmarks relies on the presence of landmarks at each point along a route where wayfinders might need assistance. The approach presented here is based on the Dutch system for navigation of cyclists. The landmarks that are used in the proposed approach are special signposts containing the necessary directional information in order to guide the wayfinder in the space. The system is quite simple, efficient and satisfactory in providing navigational assistance in indoor space. An important contribution of this research is the generation of an approach to automatically determine the decision points in indoor environments, which makes it possible to apply it to navigational assistance systems in any building. The proposed system is verified by placing numbered landmark-signs in a specific building. Several tests are performed and the results are analysed. The findings of the experiment are very promising, showing that participants reach the destinations without detours.
\end{abstract}

\section{INTRODUCTION}

In recent years, navigation has become a very active research area with a wide range of application fields. Navigation, also called finding one's way, whether in real or electronic world, is a fundamental but also complex human activity. Despite the fact that navigation systems have primarily been developed for vehicles, supporting wayfinding in indoor environments has emerged to be a significant field of interest as people spend most of their times indoors. Especially in public buildings, such as airports and train stations, hospitals, offices or university buildings, individuals often fail to find their way immediately under time pressure and would, therefore, benefit from a wellestablished system offering navigational assistance.

Without the support of external navigation aids people have to rely on common knowledge about the structure of buildings, on their previous experience and on the visual input they encounter while moving in the building (Holscher et al., 2007). However, this is a complex task. The conceptualization of wayfinding, as a spatial problem solving, incorporates information processing, decision making or planning and decision execution (Passini, 1992). Wayfinding requires explicit decision-making, such as selecting routes to follow, orienting towards non-perceptible landmarks and scheduling trips (Montello \& Sas, 2006). The sense of orientation in indoor spaces is affected by several parameters. Firstly, indoor space is characterized by the existence of the third dimension which is expressed though the different floor levels (Brunner-Friedrich \& Radoczky, 2006). Vertical movement can have a serious impact on the wayfinding performance. Moreover, indoor environments are composed of smaller, fragmented spaces, with a limited field of view and change of direction is imposed more often than outdoors. Consequently, people encounter significant difficulties in the pursuit of their destination target when they are navigating in unfamiliar indoor environments. Humans depend mainly on wayfinding directions, either provided by other people or given by maps or other wayfinding services. People use landmarks when they give route directions to anchor actions in space or to provide confirmation that the right track is still being followed (Michon \& Denis, 2001). However, while it is proven that directions provided by people are based on using landmarks as references, especially at decision points, the directions given by wayfinding services are generated based on the geometry of the space.

Empirical research has established the importance of landmarks in our understanding of and communication about space (Lynch, 1960; Michon \& Denis, 2001; Raubal \& Winter, 2002; Snowdon \& Kray, 2009). Landmark-based navigation is the most natural concept for humans to navigate themselves through their environment, especially in new and unfamiliar environments where wayfinding may be a time consuming and even challenging task, as in this type of navigation people choose a readily-identifiable feature of the general landscape and use it as a base. They can move out from the base to explore, return to the base whenever they get lost or whenever they want to start over and explore in a different direction. When they become familiar with an area, they may move from one landmark to another in order to navigate through larger spaces. It has already been proved by empirical research that landmarks simplify significantly the wayfinding task (May et al., 2003). 
This paper proposes a wayfinding assistance system based on specially designed indoor landmarks. The term landmark is used here to indicate prominent signposts, which have the potential of serving as distinctive, recognizable and salient landmarks and at the same time can provide additional information (Fontaine \& Denis, 1999; Millonig \& Schechtner, 2007). Using such signposts, a network is created, which guides the users though the building.

The remainder of this paper is structured as followed. The second section provides a background information on human wayfinding process. Section 3 discusses the original outdoor Junction Network System for cyclists, which main principles are used to build the indoor network. Section 4 presents the proposed Junction Network System for Indoor Environments. The final section discusses the validation tests and concludes on the findings.

\section{COMPONENETS OF HUMAN WAYFINDING}

Wayfinding in indoor environments is a complex process, which requires knowledge about human orientation and movement indoors as well as the type of supporting information humans need to locate themselves and find their way.

\subsection{Human orientation and navigation}

Substantial research have been completed to investigate the processes that take place when people orient themselves and navigate through space (Raubal \& Egenhofer, 1998). Several theories have been developed in order to address issues such as how people find their ways in the physical world, what they need in order to find their way, how they communicate directions and how their abilities affect wayfinding. Successful wayfinding means that a goal or destination is being reached efficiently. This requires spatial knowledge and various cognitive abilities (Raubal \& Egenhofer, 1998). McNight et al (1993) summarize the three primary ways that people employ in order to find their way: 1) landmark-navigation, in which people select easily identifiable points of reference in the environment and use them as a base, 2) route-navigation, in which they put the landmarks in a sequence creating navigation paths and they navigate in the space by learning routes between locations, and 3) mapnavigation in which people create a general frame of reference (mental/cognitive map) containing the spatial relationships between objects and use it to navigate. People's perception of the real world develops gradually through these three levels by recording information about the environment. Accordingly, for successful wayfinding information about the environment - what is in the environment and where it is - are required.

Weisman (1981) distinguished four classes of environmental variables which influence the wayfinding process: 1) visual access to familiar cues or landmarks; 2) the degree of architectural differentiation between different parts of a building; 3) use of signs or room numbers; and d) plan configuration. These variables have been examined in several studies (Weisman, 1981; O' Neill, 1991; Montello and Sas, 2006; Hölscher \& Brösamle, 2007). These studies reveal that orientation becomes difficult and wayfinding performance decreased when the visual access is restricted. However, wayfinding success increases with the presence of signage. Floor plan complexity decreases the people success in wayfinding as well. Inexperienced users prefer as much as possible central parts of the building even if it entails more detours (Hölscher et al., 2006). Users familiar with the environment use the direction strategy (following a direction) or the floor strategy (finding the floor first) in order to find their way (Hölscher \& Brösamle, 2007).

Another aspect that influence wayfinding is orientation. Orientation refers to the ability of humans to be aware of their location relative to their destination and to other places or objects (Montello \& Sas, 2006). The orientation requirements for a wayfinder can be quite coarse; it is important to know what is essential in order to reach the destination effectively and to avoid getting lost. Geographic disorientation can generate anxiety and frustration to the wayfinder. Humans can orient themselves in an environment through landmark-based and dead reckoning processes (Montello \& Sas, 2006).

Several approaches can be identified for wayfinding assistance. The most common way of navigating in indoor spaces is by presenting information about the building on maps or by signs.

Maps are the most commonly used tools in the wayfinding process. Replacing the traditional paper maps/instructions, digital maps and route descriptions are becoming wide-spread mean for wayfinding. Variety of tools have been developed, such as verbal navigational instructions, static and interactive maps, 3D visualizations and animations. While well-developed for outdoor, indoor applications are relatively limited and not well adapted to user expectations. User unfamiliar with the building or with the systems can still get lost.

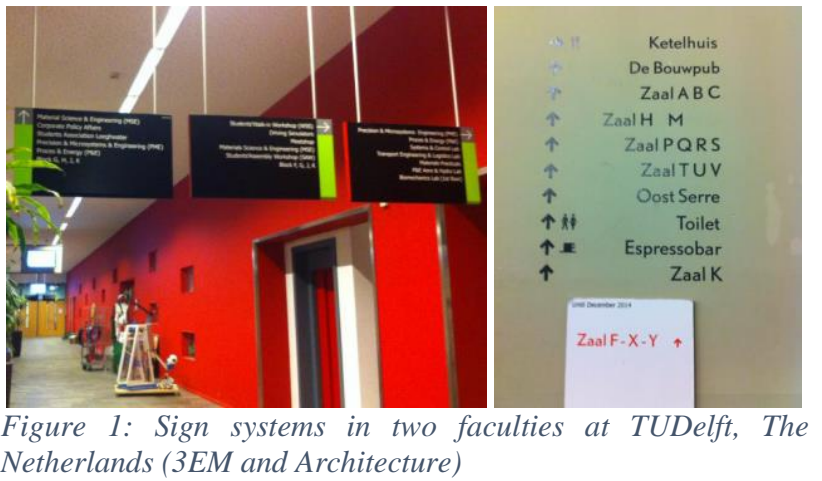

Signage is the most commonly employed physical means of enhancing wayfinding efficiency in indoor environments (Figure 1). Most building complexes offer well developed strategy for wayfinding taking into account the room names, directional signs or other graphical elements. Several studies (O' Neill, 1991; Hölscher \& Brösamle, 2007) indicate the positive relationship between signage and wayfinding performance. Signs can create confusion as well. People may have problems to understand the signs in case they are not clear or they have too much information or even confusion is generated when there are too many signs (Montello \& Sas, 2006). In this paper we argue that signs are the better approach for wayfinding indoors. Eventual difficulties with understanding and following the signs can be attributed to lack of a common reference system, i.e. language, sign nomenclature and location of the signs.

\subsection{Landmarks as source of information}

Signs are well-defined and distinguishable landmarks, which provide information about the indoor environment and the options for moving. Presson \& Montello, 1998 define landmarks as features that are relatively well-known and which define the location of other points. Landmarks are stationary, distinct, and salient objects or places, which serve as cues for structuring and 
building a mental representation of the surrounding area (Millonig \& Schechtner, 2007).

A landmark is structurally attractive if it plays a major role or has a prominent location in the structure of the spatial environment (Raubal \& Winter, 2002). From a structural point of view, landmarks can be categorized into global and local landmarks. In a wayfinding context, global landmarks are typically used for conveying directional information; they are at a distance, or off the route. Local landmarks are typically used for conveying positional information; they are close to the route, and are sometimes further categorized into landmarks at decision points, landmarks at potential decision points, and on-route landmarks along segments (Klippel et al., 2005; Lovelace et al, 1999).

Most approaches to include landmarks in wayfinding instructions focus either on landmark identification (Nothegger, Winter and Raubal, 2004; Elias, 2003) by specifying the area in which landmarks has to be sought and then identifying the features that act as outliers in the area, or on the integration of landmarks into the generated instructions (Klippel et al, 2005; Caduff \& Timpf, 2005, Ruso \& Zlatanova 2014). The failure of incorporating landmarks in commercial applications can be attributed to the costs associated with the acquisition of the required data and the highly skewed distribution of landmark candidates in available spatial data (Richter, 2013)

\subsection{Giving directions}

Route directions are important part of navigation process. These are the instructions on how to follow a route, i.e. alerting at an appropriate location (decision point) of the route which action has to be taken (Richter \& Klippel, 2005; Richter et al, 2008). The action can be related to changing the direction of movement or investigating possible options for movement or completing a task (e.g. counting doors) (Richter et al, 2008). A variety of details can be given when providing directions for wayfinding, such as landmarks, cardinal directions, street names, distances and turn descriptions (Hund \& Padgitt, 2010). Human route directions cannot make use of numerical references such as distances or angles. Instead people very often use landmarks (doors, stairs, plants) to anchor actions in space or to provide confirmation that the right track is still being followed (Michon \& Denis, 2001; Richter, 2013).

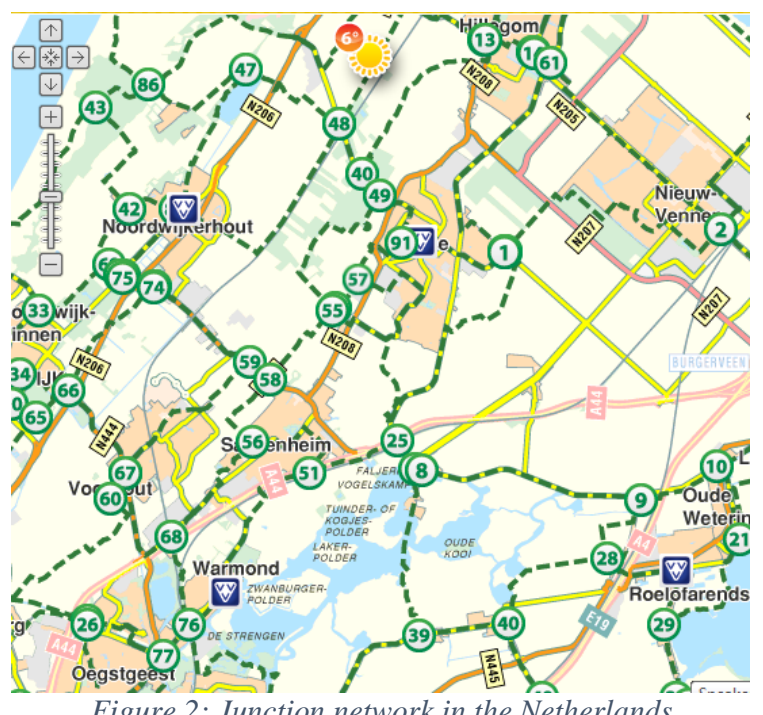

Figure 2: Junction network in the Netherlands
Specifying a direction is critical for guiding. Directional relations state the location of entities encountered along the route (like landmarks) with respect to the human or other entities; they announce a change of heading at decision points, i.e. represent turning actions; and they may relate these actions to an entity's location to better anchor them in space. In general, it can be stated that wayfinding can be characterized as following a route segment up to a decision point, making a directional choice, following the next route segment up to the next decision point, making a directional choice, and so on (Klippel et al., 2004).

\section{WAYFINDING JUNCTION SYSTEM}

\subsection{Outdoor}

The 'Junction Network System' is a special approach of providing direction instructions to cyclists, used in the Netherlands and Belgium (Figure 2). The main concept of the system is a network of nodes, which are placed at junctions of cyclist paths. The nodes are marked with a signpost (with map overview of the area) containing a unique number and directional information to the next-encountered nodes/intersections. This network has two important characteristics: 1) the number at the sign gives a unique identity to the junction and provides relative location in the space, and 2) remembering (recording) a sequence of such unique numbers allows a user-specified route to be determined. The most important principle of the system is that the assistance is provided at the point when a user has to take a decision how to go on. The signage system is a green sign with the node numbers in a white circle, which has emerged to be a special type of landmark as it is easily identified by cyclists (Figure 3 ).
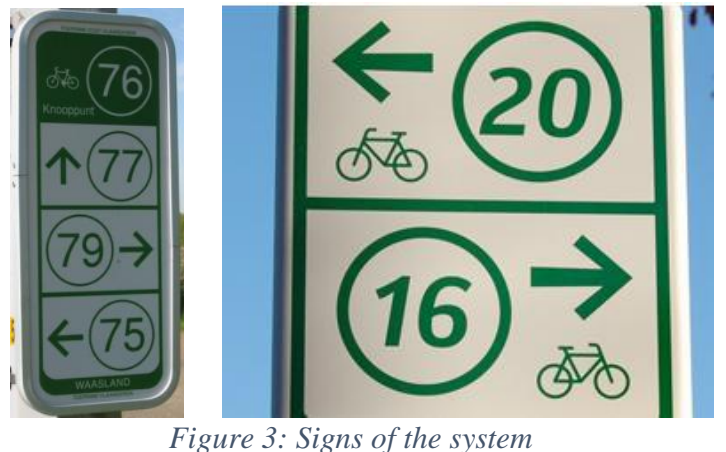

\subsection{Indoor Junction system}

The proposed system, 'Junction Network System for Indoor Environments', is the first attempt of adapting the main principles of the original 'Junction Network System' for indoor and focusing at pedestrians as a target group. The indoor concept follows closely the main principles of the original Junction Network System. The indoor variant of the system should be also able to respond to the three major questions of wayfinding communication: what information should be presented, where the information will be provided, and in what form.

Similar to outdoor, the proposed approach is based on the creation of a network of locations, equipped with a special type of signpost, the landmark-sign, containing a unique number for every location and directional information of the other numbered locations in the vicinity of it.

In this research, floor mounted signs are designed as they attract people's caution and they are well visible from a distance. Moreover, there are no important limitations regarding their size, 
they do not interfere in the configuration of space and do not intervene in people's movement (Figure 4).

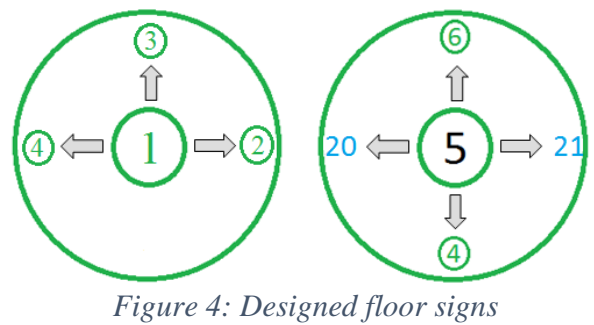

The landmark-signs are placed at decision points, where people need assistance in order to proceed further (i.e. the junctions of the original system). The location and directional information are revealed through the landmark-signs. By supplying every landmark-sign with a unique number the closest destination spaces to this sign are automatically registered to it. For example, when the user is located at number 5 , he/she can infer his relative location in the building (Figure 4, right). A directional assistance is provided by the signs that contain directional arrows indicating the closest numbered decision points or the arrival at a destination space in all possible directions of moving. The destination spaces are also marked with a unique number. It is suggested that the numbering of destination spaces (e.g. rooms) is completed after the numbering of the decision points. This facilitates following a specific route to a destination point. The location of the signs should support the conceptualization of turning instructions, enhancing the understanding of direction instructions.

The connections between the landmark-signs and the attached destinations, create the whole network of nodes which provides all possible combinations of routes between starting points and destinations. In this way, the basic representation of the system is a sequence of decision points with their accompanying actions. Guidance is given by referring to numbers of landmark-signs and anchoring actions to them. The network of landmark-signs has the same twofold role as outdoor: 1) it acts as a routing system that enhances the successfully reach of destination and 2) it plays the role of a referencing system which provides location information to the users.

There are several advantages of this system:

- It is based on an already existing and recognized system (in the Netherlands and Belgium) meaning that people can easily get used to it.

- It provides an indication of the relative position of the user in the environment. It acts not only as a wayfinding/routing system but also as a referencing system.

- The landmark-sign is well-defined object, which expresses the critical points where people need to choose direction of moving and provides location and directional information.

- It is a solution that can be applied to different building cases.

- It can be used for seamless indoor-outdoor navigation system since the principles are the same.

- $\quad$ No special devices (indoor positioning and digital maps) are required.

One of the most critical difference between indoor and outdoor junction system is locations of the landmark-signs. Outdoors the signs are placed at the junctions as they are assumed to be the decision points. Indoors, the decision points might be much more and located not only at places of crossing corridors. In contrast to outdoor, indoor environments do not have well-defines paths and humans can move freely in the open space. Therefore it is critical to decide which the possible routes are and where the landmarksigns can be placed. In this research, an automatic approach is followed to derive a network for movement and landmark-sign location placement.

The network (Figure 5) to represent the possible movement structure of the indoor environment is based on a Medial Axis Transformation (MAT) generated from Constrained Delaunay Triangulation (CDT). MAT is a good approximation of depicting the human movement in these spaces (Mortari et al., 2014). The MAT represents the connectivity of the (triangulated) spaces. Spaces are mapped to nodes of the graph, which provide access to all possible parts of the building. All building spaces are identified and represented by their central point, applying the concept of duality of spaces (Zlatanova et al, 2013). All the possible destination points and functional areas are also indicated and included in the graph (Kruminaite and Zlatanova, 2014). The connections between the spaces can represent adjacency and connectivity relationships. Connectivity ensures that humans can move further in the space. Connectivity can be established on the basis of sematic information (e.g. knowing that certain object is a door) or on the basis of geometry. For example, knowing that a floor of a room is triangulated, all the adjacent triangles have a connectivity relationship if they are not neighbouring to a nonnavigable spaces such as walls. In that way a network of nodes and edges is created which represents all the possible routes that humans can employ in a certain environment. The idea of a route skeleton corresponds to certain degree to the central-point wayfinding strategy that people usually employ in unfamiliar environments.

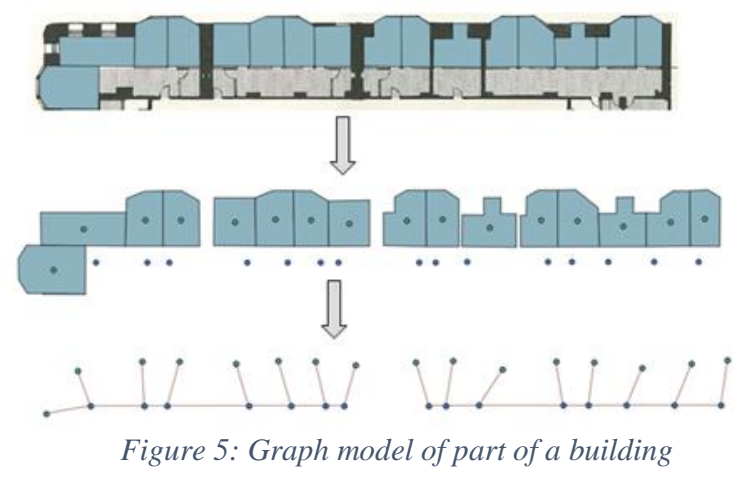

To define the location of the landmark-signs, the triangles of the CDT are investigated. Two types of generated triangular facets can be distinguished: 1) those that are built from edges that consist part of the user-specified constraints and 2) others that are free of these constraints. The last type of triangles are adjacent to other generated triangles from all three sides. Thus, humans standing at these sub-spaces are allowed to walk to three possible directions - there are no constraints to limit them to any direction. This type of generated sub-spaces corresponds in reality to areas that two or more navigable connecting spaces intersect. Consequently, in these sub-spaces decision points are located. By classifying the triangles the decision points are determined. The distinction between the two triangle types is performed based on their topological overlay relations with the originating polygon. Therefore, the topological spatial relations between the generated sub-spaces and the originating polygon are examined by using the Dimensionally Extended Nine-Intersections Model (DE9IM) (Strobl, 2008). If the result of the intersection of the boundaries of the two geometries is a point set then the triangle contains zero constrained edges. Otherwise if it results in a line set the triangle has at least one constrained edge. In that way the triangles without constrained edges are selected. 
Concluding, the steps to be followed in designing Indoor Junction system are as follows:

1. Define destinations spaces (rooms) and transition spaces (doors) of interest.

2. Determine connection spaces, i.e. the paths where movement occurs - main corridors of buildings or well-structured paths.

3. Define decision points, i.e. locations where two or more connecting spaces intersect or locations where gaps in the boundary of the connecting spaces occur.

4. Place of a landmark-sign at the decision points.

5. Provide location indication and directional information through the numbered landmark-signs.

6. Place maps of the building at the entrance and floor change points for getting overview.

7. Construct network by connecting the decision points using the triangulation analysis approach.

8. Decide on numbering approach for large multi-storey buildings, e.g. include the number of the floor in the numbering.

\section{IMPLEMENTATION}

The proposed concept was developed and tested for two buildings. This paper presents the results of the building of the Faculty of Architecture and the Built Environment, Delft University of Technology (Figure 6). The building is quite complex, contains large open spaces and elaborated existing wayfinding system. The tests were facilitated by the large number of users (students), which had the opportunity to compare the proposed with the existing wayfinding system.

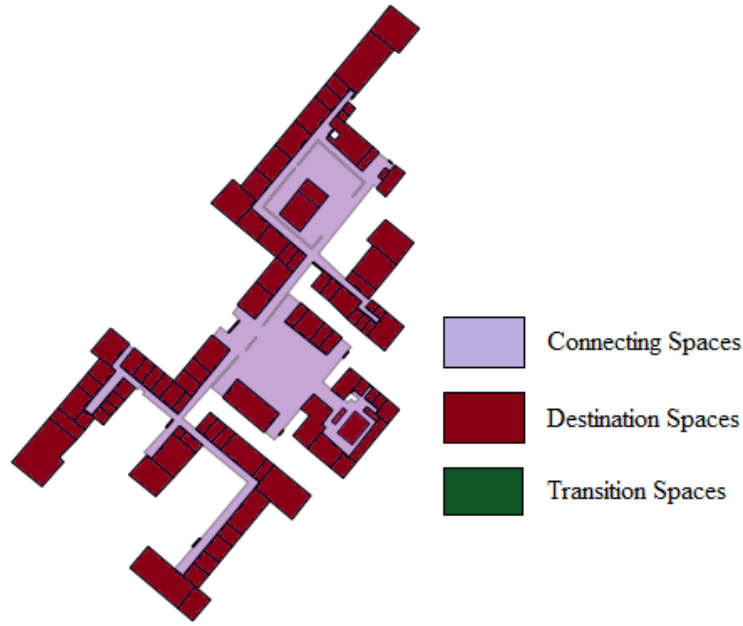

Figure 6: Identification of all spaces

The spaces of interest where identified and marked on the map of the ground floor of the building. As visible on Figure 6, the ground floor contains two large connecting spaces, which apparently require several landmark-sings.

To establish the network and the locations of the landmark-signs, the indoor environment is processed in two steps. Firstly, in the more detailed level a CDT is performed on the connecting spaces. This method subdivides the floor plan into a number of triangular-shaped non-overlapping facets, while it retains the boundary information (Figure 7). Constrained segments of the triangulation can be perceived as entities blocking the movement in the space, e.g. walls, tables, etc. Secondly, analysis of the triangulated space is performed to establish decision points and nodes for the network for human movement.
As described above, the locations of the landmark-signs is done by estimating the triangles and adjacency between them. The centroids of the triangles without constrained edges are calculated as they are the most representative points in order to act as the precise locations of decision points.

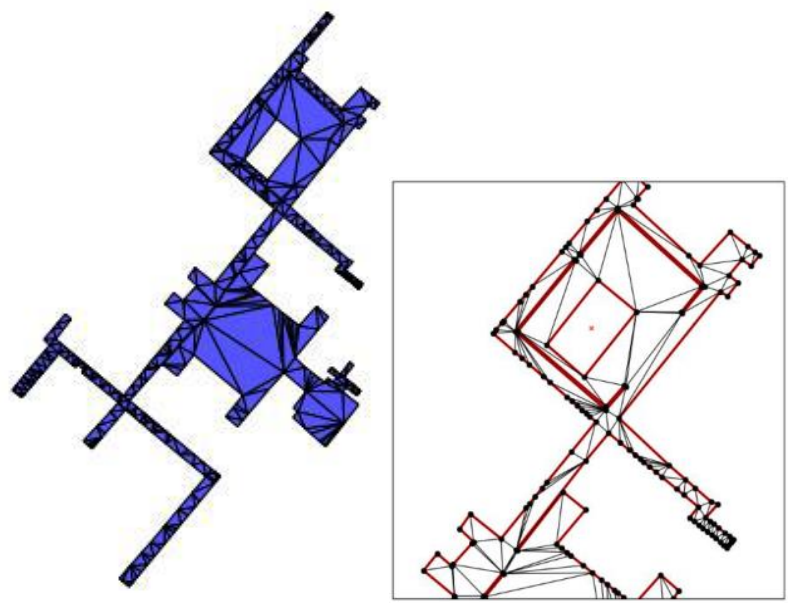

Figure 7: Constrained Delaunay Triangulation: ground floor (left) and fragment of the floor (right)

However, in some cases, some of these possible locations are very close to each other, as it is illustrated in (Figure 8). This creates redundancy of landmark-sign locations. The problem arising from this situation is that: 1) two or more signs can be installed very close to each other or even partially overlap depending on the sizes of the selected signs and 2) this entails the risk of creating confusion or misinterpretation of the assistance. Moreover, from a practical point of view, the presence of a higher number of landmark-signs requires more effort and maybe higher costs depending on the materials used for the fabrication of the landmark-signs.

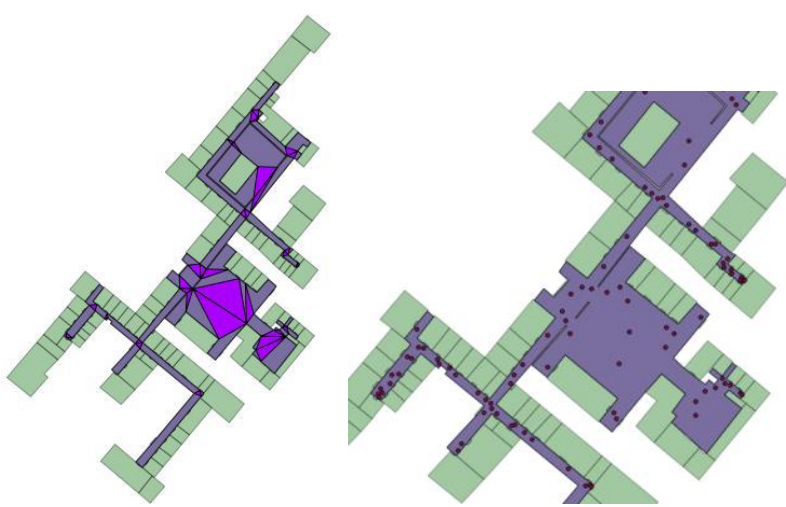

Figure 8: Spaces candidates for landmark-sign placement (left) and the final locations (right)

Another detected issue was that the locations of the landmarksigns can sometimes be quite before or even after the real location where a direction change is imposed. The cause of this circumstance is that the CDT generates large triangles with irregular shapes and small angles. In order to solve this problem, the Steiner points were inserted in order to split the constrained segments and create a more symmetrical and more regular space subdivision result (Figure 9). The Conforming Delaunay Triangulation is then used, while further area and angle constraints can be imposed to generate an even better outcome containing triangles as equilateral as possible. 

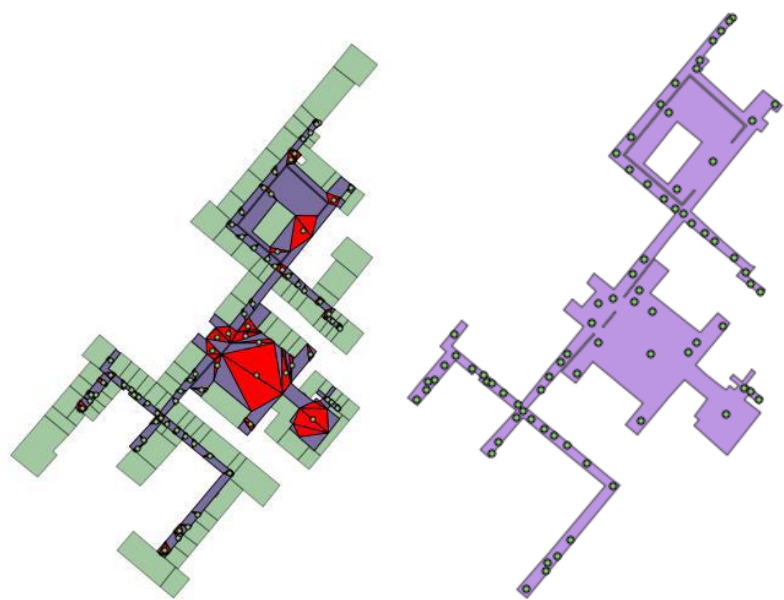

Figure 9: Final spaces (left) and locations (right) of landmarksigns.

For the generation of the network for the entire floor, the central points of polygons representing destination spaces and transition spaces are calculated and linked to the closest decision point node. In this way, all the possible routes of the building are represented and distances between the nodes can be estimated as the graph reflects not only the topological relationships between spaces but also the geometry of the building. The fact that at least three possible directions occur at the decision points is certified by the degree of the nodes. The decision points have a degree of three or more as at least three edges are incident to these vertices. All other nodes of the graph representing connecting spaces have only two links. The same is for most of the nodes representing destination spaces.

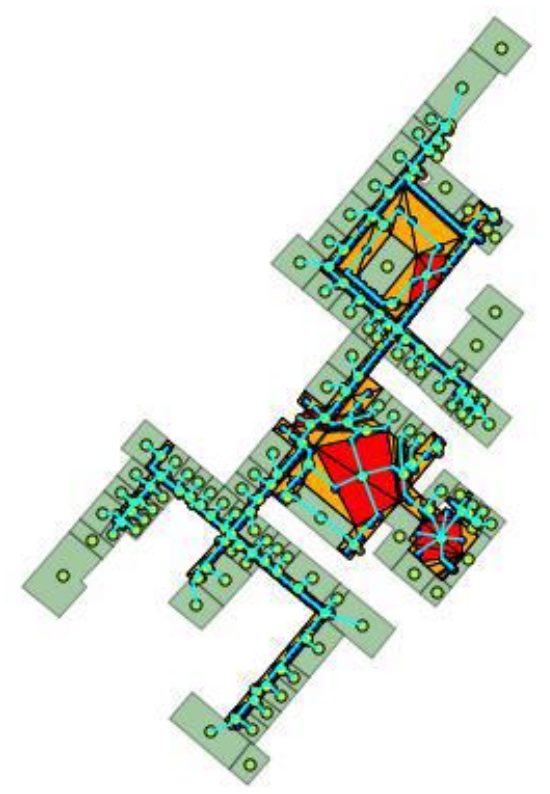

Figure 10: Final network of ground floor to be used in the Indoor Junction System

The last step, after determined all the decision points and their links in the network, is numbering the points and fabricating the landmark-signs. The number of possible directions of moving and consequently, the amount of directional arrows in the landmark-sign is derived by the degree of each one of the decision points. For the test building, the maximum degree of node encountered is four. Therefore, only landmarks-signs with three and four indications of the next numbered node were used.

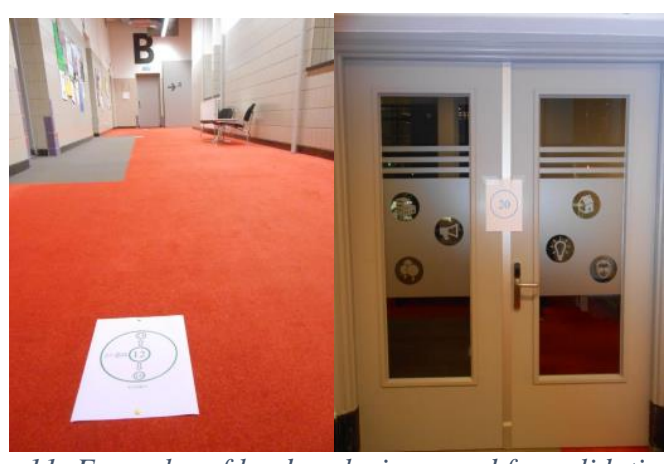

Figure 11: Examples of landmark-signs used for validation

\section{DISCUSSION}

This paper presented a new approach for indoor navigation based on a landmark-sign system, which adopts the principles of a wellknown outdoor system. The network and landmark-sign locations are automatically defined. Specific landmark-signs are proposed to indicate the decision points.

An important advantage of this system is that it provides a global solution for facilitating the wayfinding process in indoor settings. Another benefit is that the same system exists for outdoor navigation purposes, making it easier to gain people's recognition and acceptance and creating the potential of combining the two systems for providing assistance even in the case that a transition from outdoor to indoor environment is involved. Additionally, by enumerating the signposts the system can be combined not only to the outdoor Junction Network System but also with other outdoor systems based on numbers, e.g. a system numbering the individual buildings of a complex of buildings such as a university campus. Then the number of the building can be linked to the numbers of the decision points, especially to those at the entrances and exits as this is the transition area between indoor and outdoor environment.

The approach has been tested with several groups of students (familiar and not familiar with the building). They were asked to follow different routes. It was observed that the users got quicker to their destination using the proposed system, compared to the existing wayfinding system (Figure 1, right). The satisfaction with the system was quite high, the landmarks were perceived as understandable and appropriate. The feedback received after the test of the system at the Faculty of Architecture and the Built Environment reveals high peoples acceptance

The promising result of the human-based survey shows the potential of the system to act as a solution for the wayfinding indoors. People are able to follow it and that it is simple in its perception and usability. However, further testing in different building cases is definitely necessary in order to accept the system as a reliable solution.

There are also several aspects that need further attention. As mentioned above, in some cases the decision points do not coincide with the actual location where the choice of direction is imposed, so that the human should modify the automatically generated points according to the common sense during the installation phase. The main source of this error is identified at the manual work phase, where the boundaries of the polygons were digitized. Test with more buildings should further investigated how to improve the analysis of the triangulated space. 
The way of numbering the landmark-signs should be carefully decided. The numbers should follow a logical sequence, so that people to be able to remember and follow them easier, and to estimate their relative location at the building and the travelled distance. A uniform numbering system that reflects the structure of all indoor environments requires thorough investigation of the diversity of indoor space configurations.

It should be taken into consideration that in very crowded environment, detecting the landmark-signs (on the floor) from a distance might become problematic. Crowded environments were not examined. The validation test took place when the building was relatively empty. However, this problem might be overcome by the fact that the density of existing landmark-signs creates a security that humans follow the right route until they come to the next in the order landmark-sign. Nevertheless, the investigation of other locations of landmark-signs such as freestanding or ceiling-hung should be investigated as well.

Finally, one of the important conclusions of the validation tests is that some people need to be aware of the meaning of the numbers and to have an overview of the numbering system for the whole environment. This was not investigated in the context of this experiment as the focus was on testing people's ability to perceive the principles of the system. Further studies should concentrate on designing appropriate landmark-sings and a numbering system.

\section{REFERENCES}

Brunner-Friedrich, B., \& Radoczky, V. (2006). Active landmarks in indoor environments. In Visual Information and Information Systems, Springer, Berlin Heidelberg, pp. 203-215.

Caduff, D., \& Timpf, S. (2005). The Landmark Spider: Representing Landmark Knowledge for Wayfinding Tasks. In AAAI Spring Symposium: Reasoning with Mental and External Diagrams: Computational Modeling and Spatial Assistance, pp. 30-35.

Elias, B. (2003). Extracting landmarks with data mining methods. In Spatial Information Theory. Foundations of Geographic Information Science, Springer, Berlin Heidelberg, pp. 375-389

Fontaine, S., \& Denis, M. (1999). The production of route instructions in underground and urban environments. In Spatial Information Theory. Cognitive and Computational Foundations of Geographic Information Science, Springer, Berlin Heidelberg, pp. 83-94.

Hölscher, C., Meilinger, T., Vrachliotis, G., Brösamle, M., \& Knauff, M. (2006). Up the down staircase: Wayfinding strategies in multi-level buildings. Journal of Environmental Psychology, 26(4), pp.284-299.

Hölscher, C., Büchner, S. J., Brösamle, M., Meilinger, T., \& Strube, G. (2007). Signs and maps-cognitive economy in the use of external aids for indoor navigation. In Proceedings of the 29th annual cognitive science society, Austin, TX: Cognitive Science Society, pp. 377-382.

Hund, A. M., Padgitt, A.J. (210). Direction giving and following in the service of wayfinding in a complex indoor environment. Journal of Environmental Psychology, 30(4), pp. 553-564.

Klippel, A., Dewey, C., Knauff, M., Richter, K. F., Montello, D. R., Freksa, C., \& Loeliger, E. A. (2004). Direction concepts in wayfinding assistance systems. In Workshop on Artificial Intelligence in Mobile Systems, pp. 1-8.

Kruminaite, M. \& Zlatanova, S. (2014) Indoor Space Subdivision for Indoor Navigation, ISA'14, Proceedings of the Six ACM SIGSPATIAL International Workshop on Indoor Spatial Awareness, pp. 25-31.
May, A. J., Ross, T., Bayer, S. H., \& Tarkiainen, M. J. (2003). Pedestrian navigation aids: information requirements and design implications. Personal and Ubiquitous Computing, 7(6), pp. 331-338.

McKnight, C., Dillon, A. and Richardson, J. (1993). Space -- the final chapter: Or why physical representations are not semantic intentions

Michon, P. E., \& Denis, M. (2001). When and why are visual landmarks used in giving directions?. In Spatial information theory, Springer, Berlin Heidelberg, pp. 292-300.

Millonig, A., \& Schechtner, K. (2007). Developing landmark-based pedestrian-navigation systems. Intelligent Transportation Systems, IEEE Transactions on,8(1), 43-49.

Montello, D. R., \& Sas, C. (2006). Human factors of wayfinding in navigation.

Mortari, F., Zlatanova, S., Liu, L., \& Clementini, E. (2014) Improved Geometric Network Model (IGNM): A novel approach for deriving connectivity graphs for indoor navigation. ISPRS Annals of Photogrammetry, Remote Sensing and Spatial Information Sciences,4, pp. 45-51.

O'Neill, M. J. (1991). Effects of signage and floor plan configuration on wayfinding accuracy. Environment and Behavior, 23(5), 553-574.

Presson, C. C., \& Montello, D. R. (1988). Points of reference in spatial cognition: Stalking the elusive landmark*. British Journal of Developmental Psychology, 6(4), 378-381.

Raubal, M., \& Egenhofer, M. J. (1998). Comparing the complexity of wayfinding tasks in built environments. Environment and planning B, 25, pp. 895-914.

Raubal, M., \& Winter, S. (2002). Enriching wayfinding instructions with local landmarks, Springer, Berlin Heidelberg, pp. 243-259.

Richter, K. F., \& Klippel, A. (2005). A model for context-specific route directions. In Spatial Cognition IV. Reasoning, Action, Interaction, Springer, Berlin Heidelberg, pp. 58-78.

Richter, K. F., Tomko, M., \& Winter, S. (2008). A dialog-driven process of generating route directions. Computers, Environment and Urban Systems,32(3), pp. 233-245.

Richter, K. F. (2013). Prospects and Challenges of Landmarks in Navigation Services. In Cognitive and Linguistic Aspects of Geographic Space, Springer, Berlin Heidelberg, pp. 83-97.

Ruso, D., Zlatanova S. \& Clementini E. (2014) Route directions generation using visible Landmarks, ISA'14, Proceedings of the Six ACM SIGSPATIAL International Workshop on Indoor Spatial Awareness, pp. 1-8.

Snowdon, C., \& Kray, C. (2009). Exploring the use of landmarks for mobile navigation support in natural environments. In Proceedings of the 11th International Conference on Human-Computer Interaction with Mobile Devices and Services, ACM, p. 13.

Strobl, C. (2008). Dimensionally Extended Nine-Intersection Model (DE-9IM). Encyclopedia of GIS, Springer, Berlin Heidelberg, pp. 240245.

Weisman, J. (1981). Evaluating Architectural Legibility Way-Finding in the Built Environment. Environment and behaviour, 13(2), pp. 189-204.

Zlatanova, S., Liu L \& Sithole G. (2013) A Conceptual Framework of Space Subdivision for Indoor Navigation. ISA '13 Proceedings of the Fifth ACM SIGSPATIAL International Workshop on Indoor Spatial Awareness, ACM New York, NY, USA. pp. 44-48.

Lynch, K. (1960). The image of the city (Vol. 11). MIT press. 